\title{
Review
}

\section{Repair of spinal cord injuries: where are we, where are we going? ${ }^{\dagger}$}

\author{
J Fawcett*,1 \\ ${ }^{1}$ Cambridge University Centre for Brain Repair, Robinson Way, Cambridge CB2 2PY, UK
}

\begin{abstract}
Repairing the spinal cord has for a long time been a 'holy grail' for neuroscientists. No achievement in neuroscience is more difficult to achieve, and none would have the same impact amongst the medical profession and the public. Yet no patient has yet benefited from a regeneration therapy. At last sufficient progress has been made in the basic science of axon regeneration that treatments that would partially repair a spinal injury are imminent. A full repair of spinal injury still remains elusive. This review summarises progress to date, and suggests ways in which progress towards treatment of spinal injury patients might be made. Spinal Cord (2002) 40, 615-623. doi:10.1038/sj.sc.3101328
\end{abstract}

Keywords: spinal cord; axon regeneration; glia; gliosis; oligodendrocyte; trophic factors

\section{To what extent might the cord be repaired?}

Making a nervous system is the most complex part of embryogenesis, so it should not be surprising that rebuilding a damaged nervous system is very difficult. However, repair is not the same as development; the adult CNS is very much larger than that of the embryo, and there is no particular reason to believe that the response to injury will recapitulate developmental processes. Many developmental axon guidance mechanisms rely on diffusible signals that only act over short distances, so even if these molecules were appropriately expressed in the damaged CNS their effects might be very different. Some axon guidance mechanisms rely on cells that are present transiently in development but which do not reappear after injury. Many molecules that are present in the developing nervous system are not re-expressed after injury, and many molecules are present in the damaged CNS that were not present during development. For these and many other reasons the prospect of repairing spinal cord injuries by simply recapitulating development is not realistic: development and repair are not the same. Indeed at present it is not realistic to think of making a complete repair of a cord injury so as to recreate what was present before the injury. However, this does not mean that nothing useful can be achieved. If one starts looking at the problem from the other end, one can ask what would be the minimum treatment that would be useful to someone with a spinal injury. The answer is

*Correspondence: J Fawcett, Centre for Brain Repair, Robinson Way, Cambridge CB2 2PY, UK

$\dagger$ The Guttmann Lecture, International Spinal Cord Society, Vancouver, 2-6 May 2002 that for a patient with an injury in the cervical region a return of function over even one spinal segment would improve quality of life, while return of function over three or four spinal segments would transform it. Many researchers working to develop treatments for cord injury would consider that a treatment that returns function over two spinal segments would be sufficiently beneficial to be worth offering to those with cord injuries.

\section{What has to be done in order to achieve a partial repair?}

How far must the axons grow?

In order to produce a 2 spinal level improvement in motor function, corticospinal and other descending axonal pathways must regenerate over two or more spinal segments, or for around $2-3 \mathrm{~cm}$. It is possible that intraspinal circuitry below the lesion could relay information from regenerating descending axons beyond where they have regenerated. Extensive spinal relay circuits exist in rodents, but the extent to which this circuitry exists and is active in primates is controversial. In the case of ascending sensory axons, the situation is more complex. If sensory axons were required to make monosynaptic connections to the dorsal column nuclei in the medulla they would have to regenerate all the way from the lesion to that point. However, many sensory axons synapse in the dorsal horns soon after entry into the cord, so getting axons to regenerate to just above the lesion would probably be sufficient for some return of sensory function. ${ }^{1}$ 
How many axons are needed?

Many patients recover almost normal function after spinal cord injury, yet they may have lost a substantial proportion of their ascending and descending axons. In the spinal cord, as in other parts of the nervous system, it is possible to lose more than $80 \%$ of axons yet still have almost normal neurological function. The corollary also seems to be true: in various experiments in rodents, small numbers of axons have been induced to regenerate past a spinal cord injury, yet this small number of axons has returned a quite disproportionate amount of neurological function. ${ }^{-3}$

\section{Do the regenerated connections have to be accurate?} In studies in which axons have been induced to regenerate in the spinal cord and have returned some neurological function, axon tracing has usually shown evidence that the regenerating fibres have grown into spinal cord grey matter and made terminal arbors. However, the accuracy of those connections has not been examined. A reasonable assumption, taking the example of peripheral nerve regeneration, is that axons can probably recognise the right type of neurone on which to synapse but not the right spinal level. However, there are experiments in which connections that are obviously anatomically wrong can bring back useful function. For instance in rats in which one corticospinal tract has been lesioned, treatment with the IN-1 anti-Nogo antibody induces sprouting of the remaining corticospinal tract to the other side of the cord. Despite both sides of the cord being innervated by the same corticospinal tract animals make better use of the affected limbs than these control animals. ${ }^{4}$

\section{Which types of axon are needed?}

Regeneration experiments have not really addressed this issue. From knowledge of primate physiology it would be expected that some corticospinal axons would be necessary for movement control, and some of the larger sensory axons for sensation. A concern has always been that small pain fibres may regenerate better than larger sensory axons, bringing with it the danger of worsening the pain that many people with spinal injuries suffer: however, this has not been seen in experiments except where nerve growth factor, which acts on pain fibres, has been increased in the cord. ${ }^{5}$ In addition to the specific innervation from sensory and motor axons there is evidence that diffuse innvervation from serotonergic axons may be beneficial. In rats with complete spinal transections a transplant of embryonic serotonergic neurones below the injury led to extensive innvervation by small serotonergic fibres, and a considerable return of stepping and weightbearing function. ${ }^{6}$ This type of general activation could be of use in patients with partial spinal injuries who have surviving axons that do not appear to have any functional effects.

\section{Why do axons fail to regenerate in the spinal cord?}

The axons have a poor regenerative response

In order to produce regeneration in the spinal cord various problems have to be overcome. The first problem is that axons in the CNS simply do not try very hard to regenerate. There is an enormous difference between the vigour of regeneration between axons in the PNS and CNS, and between embryonic and regenerative growth. When a peripheral nerve is crushed anywhere along its length the axons rapidly make new growth cones, and the neurones quickly upregulate expression of genes, such as those for GAP-43 whose products are associated with and take part in the regenerative process. ${ }^{7,8}$ The result is rapid regeneration of the axons back to appropriate targets where they restore functional connections. Axons cut in the CNS do not respond in the same way. Where the CNS is lesioned only neurones whose axons have been cut within a few cell diameters of the cell body upregulate regeneration-associated genes, and similarly if a permissive environment such as a peripheral nerve graft is inserted into the CNS only axons that have been cut close to the cell body regenerate their axons into it. ${ }^{9}$ Even sensory axons, the peripheral branch of which regenerates vigorously when cut in the PNS, show a poor regenerative response when cut in the CNS. ${ }^{10} \mathrm{~A}$ similar difference in growth potential is seen between embryonic and adult axons. Grafts of embryonic neurones placed in the adult CNS will grow axons extensively into the host environment, yet the same type axons if cut in the adult CNS will fail to regenerate. ${ }^{11}$ The reasons for the poor regenerative response of CNS axons is not known. To some extent the difference between the regenerative response in the CNS and PNS can be ascribed to factors released by damaged peripheral nerves that stimulate regeneration, and the ability of the axon to overcome inhibitory environments depends on levels of cAMP and other signalling molecules, ${ }^{12}$ but fundamental issues remain unsolved.

\section{The CNS environment is inhibitory to regeneration}

Many different experiments have shown that the CNS environment is inhibitory to axon regeneration, and particularly the environment created by the scarring process that occurs around all CNS injuries, which is most pronounced in the spinal cord. This inhibitory nature can be ascribed to two types of inhbitory molecule; those present on myelin, and those released in the glial scar.

The first inhibitory molecule to be discovered in myelin was NogoA. This molecule, associated with the cell surface and endoplasmic reticulum of oligodendrocytes and some axons, is highly inhibitory to axon growth. It operates through one or more receptors, a Nogo receptor having recently been identified. ${ }^{13-15}$ Inhibition by Nogo involves release of calcium from internal stores within the axon. ${ }^{16}$ Many experiments, described in more detail later, have shown that 
blocking NogoA with the blocking antibody IN-1 can promote regeneration and sprouting of axons in the spinal cord and other parts of the CNS. ${ }^{17}$ Other inhibitory molecules produced by myelin are myelinassociated glycoprotein (MAG) ${ }^{18}$ and the chondroitin sulphate proteoglycan (CSPG) versican. $^{19}$ These molecules are all present in normal myelin, but are probably released in larger amounts when myelin is disrupted in injuries.

The other main source of inhibition is the glial scar that forms around CNS injuries, and is particularly marked after spinal cord injury. The glial scarring process is complex, and involves astrocytes, oligodendrocyte precursors, meningeal cells and microglia. The main inhibitory molecules produced in these structures are CSPGs, which made by all these cell types except microglia. ${ }^{20}$ The CSPGs upregulated in CNS injuries are NG2, neurocan, versican, and phosphacan made by oligodendrocyte precursors, neurocan, phosphacan and brevican made by astrocytes, and NG2 and versican made by invading meningeal cells. ${ }^{21-23}$ All of these CSPGs are inhibitory to axon growth. The mechanism by which the CSPGs inhibit growth is complex. Neurocan and phosphacan can associate directly with cell surface adhesion molecules to block their ability to promote axon growth. However, much of the inhibitory activity of the molecules depends on the glycosaminoglycan (GAG) sulphated sugar chains attached to them. ${ }^{24}$ The probable mechanism is that the protein core of the CSPG attaches to a matrix or cell surface molecule, bringing the highly charged GAG chain into a position in which it can mask or alter the structure of the target molecule, thereby abolishing its ability to promote axon growth. Treatments that remove the GAG chains or intefere with their synthesis can promote the growth of axons through glial scars both in vitro and in vivo. ${ }^{1,25}$

The final issue to consider is the nature of the spinal injury itself. Only one-third of these in humans completely transect the cord. Human spinal injuries are large, with extensive scarring and meningeal invasion. For many types of injury some form of bridge will be needed to take regenerating axons across the injury. ${ }^{26}$

\section{What types of treatment promote spinal cord repair?}

From the preceding section it can be seen that treatments that would be expected to induce axon regeneration would be aimed at (1). Increasing the regenerative vigour of cut axons, (2). Replacing the inhibitory CNS environment with something more permissive, or bridging a spinal injury with the same, (3). Neutralising the inhibitory effects of the myelinrelated inhibitory molecules NogoA, MAG and versican, (4). Neutralising the inhibitory effects of the glial scar-related CSPGs NG2, neurocan, versican, brevican and phosphacan. All of these strategies have been attempted and all have produced significant regeneration in rodent spinal injury models.
(1) Increasing the regenerative vigour of cut axons

Many in vitro experiments have shown that neurotrophins are able to increase the regenerative vigour of axons, and these results have been confirmed in regeneration experiments in vivo. Different axons have different neurotrophin receptors, so each neurotrophin acts on particular groups of axons. ${ }^{27}$ Neurotrophins have been applied either by direct infusion or injection, by viral transduction or by inserting neurotrophinsecreting cells into the cord. Direct application of purified neurotrophins has been successful in inducing axon regeneration in the cord by itself, ${ }^{28}$ or larger amounts of regeneration has been achieved by combining neurotrophin treatment with either anti Nogo antibodies or embryonic grafts. ${ }^{3,29}$ The most successful neurotrophin has been NT-3, for which there are receptors on corticospinal and on large sensory axons, and corticospinal axons regenerate in large numbers past grafts of NT-3 secreting fibroblasts. ${ }^{30}$ Nerve growth factor also promotes regeneration, but receptors for it are prominent on small pain axons, so hyperalgesia can be caused by its presence in excess. ${ }^{5}$ An alternative to the application of neurotrophins is to apply treatments that have direct effects on axonal signalling pathways. There is evidence that the positive effects of neurotrophins are mediated via cyclic nucleotides, and that inhibitory molecules in the environment act via the small GTPase Rho. Blocking Rho has been successful at promoting regeneration in the spinal cord and optic nerve, ${ }^{31,32}$ and increasing levels of cAMP also promotes axon regeneration. ${ }^{12}$ One of the issues affecting the regenerative response of axons that is not yet understood is the difference in response when axons are cut close to the cell body or further away. In general, CNS axons regenerate more successfully if they are cut close to their cell body. Since many of the important axons that are damaged following a spinal cord injury are cut far from their cell body, this is an important issue. An improved understanding of the factors that govern the vigour of the axonal regenerative response will be crucial if we are to progress to greater amounts of axon regeneration than have been achieved so far.

\section{(2) Replacing the inhibitory CNS environment with a permissive environment}

Since axons regenerate successfully in the Schwann cell environment of peripheral nerves but not in the environment of the damaged CNS, it makes sense to place Schwann cells or peripheral nerve grafts into the CNS to promote regeneration. This was first done by Tello and Cajal at the turn of the century, and the approach was revived by David and Aguayo. ${ }^{33}$ Since then many types of peripheral nerve or Schwann cell grafting experiments have been performed. Damaged axons regenerate close to Schwann cell containing grafts, and their axons are attracted into the Schwann cell environment. Once in the grafts, axons have been seen to regenerate for considerable distances. ${ }^{34,35}$ 
However, the Schwann cell environment is so attractive that axons are unwilling to leave the grafts to re-enter the CNS environment. This problem is exacerbated by the fact that astrocytes and Schwann cells do not mix, so there is a distinct graft-host boundary which axons can only cross in the host to graft direction. This problem is less serious when the end of the Schwann cell containing graft is very close to neurones with which the regenerating axons can form connections. Thus, when grafts are placed in the optic nerve to take regenerating optic axons to the neuropil of the superior colliculus, significant numbers of axons were able to leave the grafts to form functional connections, and substantia nigra axons can leave a Schwann cell environment to enter their target area in the striatum. ${ }^{36,37}$ In the injured spinal cord, regenerating axons need to grow beyond the graft before synapsing, so the Schwann cell astrocyte boundary problem makes peripheral nerve and Schwann cell grafts of little practical use. Various attempts have been made to persuade axons to leave the grafts and re-enter the CNS environment, such as injecting neurotrophins, but these have only been partially successful. ${ }^{38}$ More promising have been grafts containing olfactory glia. In the normal adult CNS, olfactory axons are constantly growing into the CNS from new neurones in the olfactory mucosa, and these axons enter the CNS in contact with specialised olfactory ensheathing cells. Because these cells enable axons to enter the CNS they have been used as grafts to repair the damaged spinal cord. Various repair experiments have been performed using these cells. In some experiments the results have been dramatically successful. The olfactory glia, unlike Schwann cells, are able to migrate into the host CNS environment and through the glial scar region surrounding a spinal cord injury, carrying regenerating axons with them (Figure 1e). These axons have then been able to grow on beyond the olfactory glia to form connections in the host spinal cord which have mediated a return of function. ${ }^{39-41}$ Olfactory glia have also been placed at the interface between Schwann cell grafts and host CNS, and have allowed axons to leave the Schwann cell environment to reenter the CNS environment. ${ }^{42}$ However, some repair experiments with olfactory glia have been less successful. In general in these experiments highly purified olfactory ensheathing cells have been used, while in the successful repair experiments a mixture of olfactory glia were grafted. There is some evidence that the different types of olfactory glia work together, and this may be important in allowing them to migrate in the CNS and promote regeneration. The third type of tissue that has been inplanted into the CNS is embryonic spinal cord. This tissue is also permissive to axon regeneration, and implantation into the injured cord produces some return of function, which is much greater if neurotrophis are given at the same time. ${ }^{3}$ Exactly how the grafts exert their effect is not completely clear. Host axons regenerate into embryonic grafts and appear to form connections with the neurones in the graft, but only in the presence of exogenous neurotrophins will axons regenerate through the grafts and back into the host cord (Figure 1d). However, the neurones of the embryonic graft certainly grow axons into the host cord for some distance. Much of the action of the embryonic grafts is probably, therefore, as a relay, with host axons connecting to graft neurones, and graft neurones sending their axons to connect with host cord neurones.

\section{(3) Neutralising the inhibitory effects of myelin molecules}

The identified myelin inhibitory molecules are NogoA, MAG, tenascin-R and versican. Of these, only NogoA has had specific treatments aimed at it. One of the turning points of regeneration research was the development of the IN-1 antibody which blocks the inhibitory action of NogoA. This antibody was applied to rat spinal cord injuries by implanting antibodysecreting hybridoma cells into the brain. The corticospinal tract was labelled, and axons were seen regenerating for distances of over $1 \mathrm{~cm}^{43}$ (Figure 1a). Behavioural assessment showed that some spinal cord functions that rely on corticospinal function returned in these animals. Since that time the actions of the IN-1 antibody have been examined in many axon pathways, and almost all of them have shown some regeneration. In addition a co-operative effect exists between the IN-1 antibody and the neurotrophins NT-3 and BDNF. Thus, when rat spinal injuries were treated with a combination of IN-1 and NT-3 larger numbers of axons regenerated for a greater distance than with either treatment alone ${ }^{29}$ (Figure 1a). In addition to its actions on axon regeneration IN-1 causes sprouting of various axons. In the spinal cord, when one corticospinal tract was cut the other corticospinal tract sprouted axons to the other side of the cord in the presence of $\mathrm{IN}-1$, and this was associated with return of various motor functions. ${ }^{44,45}$ A more general blockade of myelin molecules has been achieved by immunising mice with myelin, or transferring antibodies from myelin-immunised animals. This strategy presumably leads to the development of antibodies to many molecules. Animals treated in this way show robust regeneration in the injured spinal cord. ${ }^{46}$

\section{Neutralising inhibitory molecules in the glial scar}

The main inhibitory molecules in the glial scar are chondroitin sulphate proteoglycans (CSPGs). Because several of these molecules are present in the injured CNS, it is unlikely that neutralisation of a single CSPG would be effective in promoting axon regeneration. However, all CSPGs share commmon features, the most important being that at least part of their inhibitory properties rely on the glycosaminoglycan (GAG) chains made up of sulphated sugars. Some bacteria have evolved enzymes to digest these GAGs, presumably to enable them to penetrate scar tissue 
(Figure 1b). Chondroitinase has been shown to promote axon regeneration through CNS glia in vitro ${ }^{24}$ and in vivo, ${ }^{25}$ and has now been applied to rat spinal cord injuries. When infused directly into a partial cord lesion involving the dorsal columns, chondroitinase promoted regeneration of both sensory and motor axons, and considerable return of function ${ }^{1}$ (Figure 1b). There are reasons to believe that some of the return of function is due to an enhancement of plasticity within the cord.

\section{State of the art in promoting axon regeneration in the injured cord}

As recounted above there are now several treatments that can promote limited regeneration in rat spinal injury models. The maximum length of regeneration to date is around $4 \mathrm{~cm}$, but in most experiments axons regenerate for $1-2 \mathrm{~cm}$. Because the various treatments act via very different mechanisms, it is tempting to believe that they would have additive effects. This has been shown to be the case with the IN-1 antibody and NT-3, but other combinatorial treatments have not been assessed. Despite the great advances made in obtaining regeneration in the past decade, there is at present no experimental evidence to suggest that axons can be made to regenerate for more than about $4 \mathrm{~cm}$. This is a much smaller distance than is seen in peripheral nerve regeneration. In order to increase CNS regeneration it will be necessary to take further steps to make the CNS environment more permissive, but the major need is probably to increase the regenerative response of CNS axons.

\section{Promoting the function of unlesioned axons}

Two-thirds of human cord injuries are anatomically incomplete, with axons passing across them. In principle it should be easier to enhance the function of these than it is to promote regeneration. However, there has been surprisingly little work done on this avenue. There are two main areas of research that are relevant to this issue. The first is demyelination/ remyelination. After a cord injury there is widespread demyelination, and many of the surviving axons in the region of the injury lack myelin. ${ }^{47}$ This may be one reason why they would fail to conduct and promote function. It is not clear if these axons spontaneously remyelinate. In most spinal injuries there is extensive Schwann cell invasion with remyelination, and oligodendrocyte precursors respond vigorously to spinal cord injury. Several years after injury there are few if any remaining demyelinated axons. It is not clear whether this is because they have all remyelinated or because the demyelinated axons have died, as happens in multiple sclerosis. There are various potential methods for remyelination being developed in the context of multiple sclerosis. Readers can refer to reviews on this subject. ${ }^{48}$ The second possible way of promoting the function of surviving axons is via plasticity. In the adult CNS after damage function can be restored to some extent by the unmasking of silent connections, and by the formation of new connections. Within the normal CNS many synaptic connections are silent, their function being suppressed by inhibitory mechanisms. After damage, inhibition may be lifted so that these silent synapses become active. This can happen rapidly, and has been shown to bring back some function after cortical injury. ${ }^{49,50}$ Although axons cannot regenerate in the adult mammalian CNS, sprouting of new connections can occur at the nerve terminals. When synaptic space is vacated by damage to axons and neurones, the surviving processes can sprout new terminals and form new synapses (reviewed in ${ }^{51}$ ). This process is slow, and again has been shown to be associated with return of function. However, some forms of plasticity are probably maladaptive. Three of the treatments described above for promotion of axon regeneration also promote plasticity. The anti NogoA antibody IN-1 has been applied to a number of spinal cord injury models. It shows an impressive ability to promote sprouting of the corticospinal and other tracts. Thus, when the corticospinal tract on one side of a rat was cut, in the presence of IN-1 the axons of the remaining tract sprouted across the midline to innervate the contralateral cord, and also contralateral nuclei within the hind and midbrain. This was associated with considerable return of function, demonstrating that inappropriate connections within the cord can bring back significant useful function. ${ }^{4}$ The inhibitory CSPGs appear to restrict plasticity in the adult CNS. Shortly after birth there is a critical period when the brain and cord show great plasticity, but this stops early in life. The main mechanism for stopping this plasticity is probably the laying down of jackets of CSPG around neurones, and this is seen particularly in the cord. Digestion of these structures with the enzyme chondroitinase has recently been shown to reactivate plasticity in the cortex (Pizzorusso et al. unpublished results) and also probably in the cord. ${ }^{1}$ The third potential method for enhancing plasticity is through the use of neurotrophins. Treatment of the cord with neurotrophins produces local sprouting of axons with functional effects. ${ }^{5,52,53}$ One of these effects is not helpful, which is NGF-induced sprouting of pain fibres, leading to hyperalgesia. Overall, despite the fact that there are now treatments that can influence plasticity in the cord, there remains a need to establish whether these treatments could be helpful in animal models of partial cord damage, and therefore to patients with partial lesions of the cord.

\section{Translating experimental results towards treatments for human cord injuries}

The first step in producing a treatment for human cord injuries is the development of experimental methods that can promote regeneration and plasticity. As described in the preceding paragraphs, basic research 


\section{a}

The IN-1 anti NogoA antibody promotes regeneration after $\mathrm{SCl}$



lesion tract and treat with Schwab IN-1 antibody some regeneration with functional effects

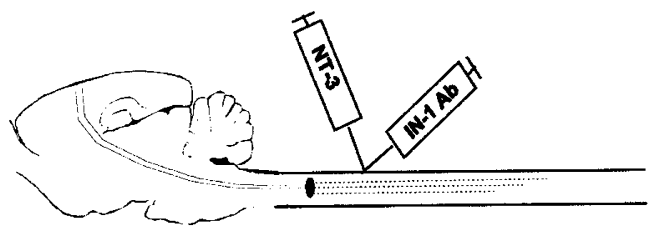

C

NT-3 secreting fibroblasts promote regeneration of corticospinal axons

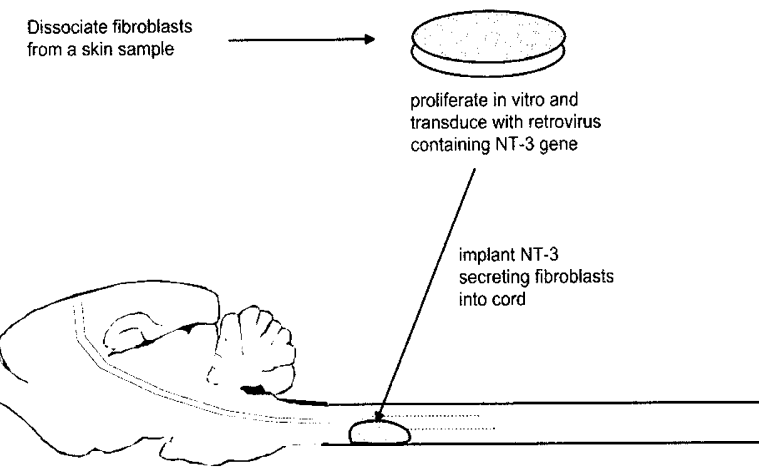

b

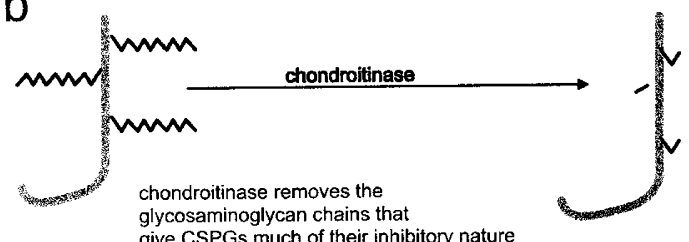
give CSPGs much of their inhibitory nature

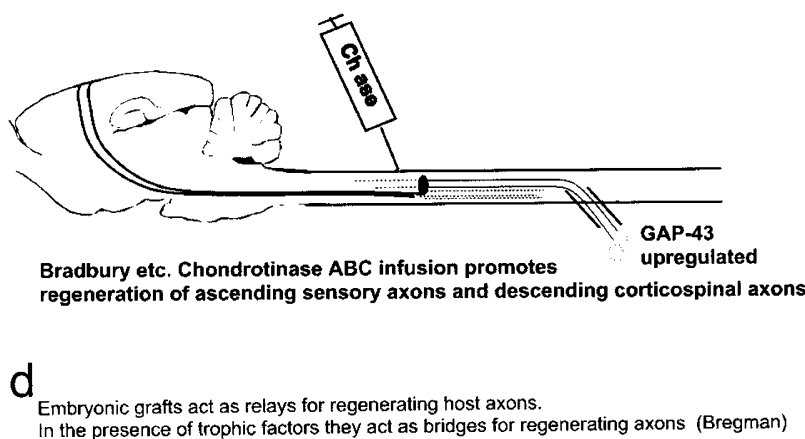

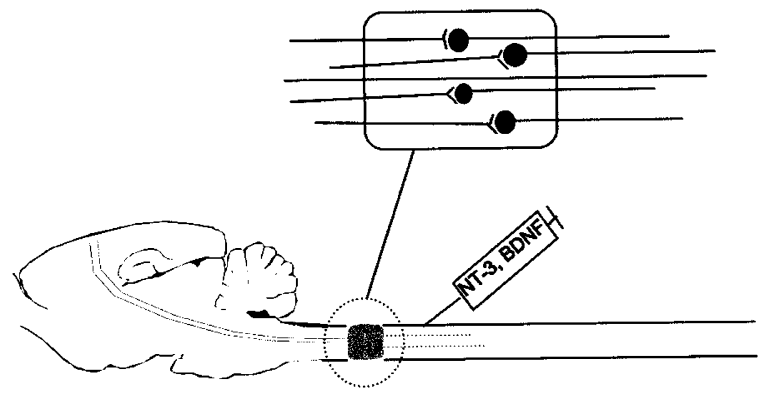



Figure 1 Five treatments that have been successful in promoting significant amounts of axon regeneration in rodent spinal injury models. (a) The monoclonal anti Nogo A antibody IN-1 promotes regeneration of corticospinal and other axons and axonal sprouting. Its efficacy is increased when combined with NT-3. The treatment promotes considerable functional recovery. (b) Chondroitinase $\mathrm{ABC}$ removes the glycosaminoglycan chains from chondroitin sulphate proteoglycans, leaving behind a small four sugar stub. This removes most of the inhibitory activity from the molecules. Applied in vivo to spinal cord injuries chondrotinase promotes regeneration of both sensory and motor axons and functional recovery. (c) Fibroblasts from the skin of individual animals or patients may be grown and proliferated in tissue culture, then transplanted back. This removes the risk of immune rejection. The cells may be transduced with a viral vector while in culture, to secrete a trophic factor. NT-3 secreting fibroblasts implanted into the cord promote axon regeneration and functional recovery. (d) Grafts of embryonic spinal cord 
has now produced a range of methods to do this. The amount of regeneration and plasticity that they can produce is not sufficient to produce a complete repair of a spinal cord injury, but is probably sufficient to make a discernable improvement to the neurological function of a patient with spinal injury. Moreover, progress in this area is sufficiently rapid that increasing amounts of regeneration will almost certainly be achieved in the next few years. The current challenge, therefore, is to apply these treatments to humans with spinal cord injuries. What steps are needed in order to move from experimental studies in rats to treatment of humans? Two processes need to proceed in parallel. The first is verification of the various experimental treatments in more lifelike models of spinal injury, the second is the preparation of clinical trials protocols.

\section{Verification of experimental treatments in lifelike models of spinal injury}

Most regeneration experiments have been performed using partial scissor or knife cuts of the cord. This type of lesion is good for quantification of axon regeneration and very repeatable, but not very similar to a human cord injury. Some experiments have been performed using various types of impactor, which produce a much more widespread injury that more closely resembles a human injury. However, all rodent experiments are done in a cord which is very much smaller than that of humans. In order to move forwards it is necessary to verify our current experimental treatments both in impactor injuries in rodents, and in larger animals. Moreover, many experimental treatments are applied at the time of injury. The first trials of treatments in patients may need to be performed in chronically injured patients, and of course there are millions of such patients around the world who would hope one day to be treated. There is, therefore, a need to find out which of the various treatments can produce axon regeneration in spinal cords that have been injured weeks or months previously. Experiments such as these have been performed and are in progress for transplants of trophic factor secreting cells, and have shown regeneration, although not quite so vigorous as regeneration immediately after injury. ${ }^{54}$ The choice of a large animal model in which to verify treatments remains controversial. The choice is between dog, cat and monkey. The dog model has several advantages. Spontaneous dog spinal injury is common-large veterinary practices see several a week - and owners are often helpful in allowing their pets to receive experimental treatment. However, the injuries are uncontrolled and therefore variable, and the amount of data that can be gleaned is limited. A trial of ensheathing cell transplantation in spontaneous dog injuries is about to start. The cat offers two main advantages. First, it tolerates spinal lesions well, and second, our knowledge of cat spinal physiology is very good. However, there are important differences between the human and cat spinal cord in respect of the motor circuitry and the glial responses to injury. Primates would be the most convincing large animal model for verification of treatments. However, the practical difficulties are formidable. Primates tolerate complete spinal injuries poorly and are difficult to care for, although partial spinal injury is better tolerated in some species. Animal care considerations mean that it is not acceptable to injure large numbers of primates for open ended experiments, and the cost and logistics are prohibitive. However, trials of the IN1 anti Nogo antibody are being performed in a small number of primates. The answer may turn out to be a combination of large animal trials. The first may be performed in dog or cat, perhaps with final verification in a very small number of primates.

\section{Preparations for trials of treatments in patients}

Given that the amount of repair that will be achieved with current treatments is limited, it is crucial to have in place clinical protocols that can show unequivocally whether a treatment has produced benefit, no change, or made a patient worse. Current clinical assessment protocols are adequate for determining the function of a patient from the perspective of organising their rehabilitiation, but lack the fine grain and objectivity needed for a good clinical trial. Our knowledge of the precise physiology of the injured human spinal cord is quite limited, so the preparation of robust trial protocols will take some time. In preparing such protocols the first consideration must be what type of patient will be treated. Will they be freshly or chronically injured, what level will the injury be, and will they have complete, incomplete or dyscomplete lesions? The first consideration must be safety. It is not acceptable to take the risk of making any patient worse. Since any experimental intervention must carry risk it is therefore reasonable to target the first treatments to patients with lesions in the thoracic region, where even if the treatment produces additional damage the patient will suffer only marginal functional consequences. Making a C5 patient into a $\mathrm{C} 4$ patient would be

tissue to bridge complete lesions of the cord promote regeneration of host axons into the graft to synapse with graft neurones, the host neurones sending their axons to synapse in host spinal cord. In the presence of trophic factors axons may also grow straight through the graft and back into host spinal cord. The grafts act partly as a bridge, partly as a relay. (e) Olfactory glia from the olfactory bulb can be grown in tissue culture and proliferated. When transplanted back into a spinal cord injury the cells migrate from their implantation site, carrying regenerating axons with them. These regenerating axons bring substantial functional recovery 
disastrous, but the functional difference between a T8 and T7 lesion is small. However, there are disadvantages to thoracic lesions. Many patients with such lesions have widespread and severe injuries to their bodies. Some parts of the thoracic cord have a very vulnerable blood supply. Assessment of functional improvement or deterioration is difficult. The prudent course is probably to carry out the first trials of treatment in thoracic lesions, moving quickly to higher lesions if the initial trials show that it is safe to do so. The next issue is whether to do the first trials on acute or chronic injuries. The advantage of acute injuries is that the regenerative potential of the axons is probably at its highest at this time, and the possibilities of modifying the lesion environment to make it more permissive are greatest. However, this has to be set against the disadvantage that the eventual outcome of spinal injuries is usually somewhat uncertain, making it difficult to decide whether the treatment has worked, and the patients often have many severe problems, which means that the priority is to preserve life rather than making a trial of treatment of unknown efficacy. Treatment of chronic lesions is much less problematic, but axotomised neurones may have atrophied or died, and a dense scar tissue is in place at the injury site. However, the condition of the patient will have stabilised, so each patient can be their own control. Probably the best initial course is to intervene as soon as the patient's medical condition has stabilised and the outcome of the spinal injury is predictable, which means three weeks or more after injury, progressing to more acute lesions as experience accumulates. The last difficult question is which treatment to try first. The best treatment will probably be a combination of the various ones described earlier: for instance a combination of the IN-1 antibody, chondroitinase and a neurotrophin. However, it will not be possible to apply all three together in the first trial patients. The various national legislative organisations will insist that each treatment is applied by itself in the first instance to demonstrate its safety. Only after it is clear that individual treatments are safe will it be possible to attempt combination therapies. In regulatory terms, the most advanced treatment is probably IN-1, so this will probably be the first treatment offered to spinal injury patients.

\section{Conclusion}

Repairing spinal injuries is the most difficult thing that medical science has ever attempted. Basic science has now produced treatments that will probably benefit humans with spinal injuries, but which fall far short of offering full repair. Translating these experimental therapies to human patients is enormously challenging, and will involve well integrated groups of basic and clinical scientists working closely together to cross almost entirely uncharted territory. However, the fact that we have reached this point is in itself an historic moment.

\section{References}

1 Bradbury EJ et al. Chondroitinase ABC promotes axon regeneration and functional recovery following spinal cord injury. Nature 2002; 416: 636-640.

2 Merkler D et al. Locomotor recovery in spinal cordinjured rats treated with an antibody neutralizing the myelin-associated neurite growth inhibitor Nogo-A. $J$ Neurosci 2001; 21: 3665-3673.

3 Coumans JVet al. Axonal regeneration and functional recovery after complete spinal cord transection in rats by delayed treatment with transplants and neurotrophins. $J$ Neurosci 2001; 21: $9334-9344$.

4 Raineteau O, Schwab ME. Plasticity of motor systems after incomplete spinal cord injury. Nat Rev Neurosci 2001; 2: 263-273.

5 Romero MI, Rangappa N, Li L, Lightfoot E, Garry MG, Smith GM. Extensive sprouting of sensory afferents and hyperalgesia induced by conditional expression of nerve growth factor in the adult spinal cord. J Neurosci 2000; 20: $4435-4445$.

6 Ribotta MG et al. Activation of locomotion in adult chronic spinal rats is achieved by transplantation of embryonic raphe cells reinnervating a precise lumbar level. J Neurosci 2000; 20: $5144-5152$.

7 Plunet W, Kwon BK, Tetzlaff W. Promoting axonal regeneration in the central nervous system by enhancing the cell body response to axotomy. J Neurosci Res 2002; 68: $1-6$.

8 Caroni P. Intrinsic neuronal determinants that promote axonal sprouting and elongation. Bioessays 1997; 19: $767-775$.

9 Tetzlaff W et al. Response of rubrospinal and corticospinal neurons to injury and neurotrophins. Progress In Brain Research 1994; 103: 271 - 286.

10 Chong MS et al. Intrinsic versus extrinsic factors in determining the regeneration of the central processes of rat dorsal root ganglion neurons: the influence of a periperal nerve graft. J Comp Neurol 1996; 370: $97-104$.

11 Fawcett JW. Intrinsic control of regeneration and the loss of regenerative ability in development. In: Ingoglia $\mathrm{N}$, Murray M (eds). Nerve Regeneration. New York: Marcel Dekker, 2000.

12 Cai D et al. Neuronal cyclic amp controls the developmental loss in ability of axons to regenerate. $J$ Neurosci 2001; 21: 4731 - 4739 .

13 Bandtlow CE, Schwab ME. NI-35/250/nogo-a: a neurite growth inhibitor restricting structural plasticity and regeneration of nerve fibers in the adult vertebrate CNS. Glia 2000; 29: 175-181.

14 Fournier AE, Grandpre T, Strittmatter SM. Identification of a receptor mediating Nogo-66 inhibition of axonal regeneration. Nature 2001; 409: $341-346$.

15 Grandpre T, Nakamura F, Vartanian T, Strittmatter SM. Identification of the Nogo inhibitor of axon regeneration as a Reticulon protein. Nature 2000; 403: 439-444.

16 Bandtlow CE et al. Role of intracellular calcium in NI35 -evoked collapse of neuronal growth cones. Science 1993; 259: $80-83$.

17 Schwab ME. Repairing the injured spinal cord. Science 2002; 295: 1029-1031.

18 Mukhopadhyay $\mathrm{G}$ et al. A novel role for myelinassociated glycoprotein as an inhibitor of axonal regeneration. Neuron 1994; 13: $757-767$. 
19 Niederost BP, Zimmermann DR, Schwab ME, Bandtlow CE. Bovine CNS myelin contains neurite growthinhibitory activity associated with chondroitin sulfate proteoglycans. J Neurosci 1999; 19: 8979-8989.

20 Fawcett JW, Asher RA. The glial scar and CNS repair. Brain Res Bull 1999; 49: 377 - 391.

21 Asher RA et al. Versican is upregulated following CNS injury and is a product of oligodendrocyte lineage cells. $J$ Neurosci 2002; 22: $2225-2236$.

22 Asher RA et al. Neurocan is upregulated in injured brain and in cytokine-treated astrocytes. J Neurosci 2000; 20: $2427-2438$

23 Levine JM, Reynolds R, Fawcett JW. The oligodendrocyte precursor cell in health and disease. Trends Neurosci 2001; 24: 39-47.

24 Smith-Thomas L et al. Increased axon regeneration in astrocytes grown in the presence of proteoglycan synthesis inhibitors. J Cell Sci 1995; 108: 1307-1315.

25 Moon LDF, Asher RA, Rhodes KE, Fawcett JW. Regeneration of CNS axons back to their original target following treatment of adult rat brain with chondroitinase ABC. Nat Neurosci 2001; 4: 465-466.

26 Geller HM, Fawcett JW. Building a bridge: engineering spinal cord repair. Exp Neurol 2002; 174: 125-136.

27 Jones LL, Oudega M, Bunge MB, Tuszynski MH. Neurotrophic factors, cellular bridges and gene therapy for spinal cord injury. $J$ Physiol 2001; 533: 83-89.

28 Bradbury EJ et al. NT-3 promotes growth of lesioned adult rat sensory axons ascending in the dorsal columns of the spinal cord. Eur J Neurosci 1999; 11: 3873-3883.

29 Schnell L et al. Neurotrophin-3 enhances sprouting of corticospinal tract during development and after adult spinal cord lesion [see comments]. Nature 1994; 367(6459): $170-173$.

30 Grill R, Murai K, Blesch A, Gage FH, Tuszynski MH. Cellular delivery of neurotrophin-3 promotes corticospinal axonal growth and partial functional recovery after spinal cord injury. J Neurosci 1997; 17: 5560 - 5572.

31 McKerracher L. Spinal cord repair: strategies to promote axon regeneration. Neurobiol Dis 2001; 8: $11-18$.

32 Lehmann $\mathrm{M}$ et al. Inactivation of Rho signaling pathway promotes CNS axon regeneration. J Neurosci 1999; 19: $7537-7547$

33 David S, Aguayo AJ. Axonal elongation into peripheral nervous system bridges after central nervous system injury in adult rats. Science 1981; 241: 931 -933.

34 Aviles-Trigueros M, Sauve Y, Lund RD, Vidal-Sanz M. Selective innervation of retinorecipient brainstem nuclei by retinal ganglion cell axons regenerating through peripheral nerve grafts in adult rats. J Neurosci 2000; 20: $361-374$.

$35 \mathrm{Xu}$ XM et al. Bridging Schwann cell transplants promote axonal regeneration from both the rostral and caudal stumps of transected adult rat spinal cord. J Neurocytol 1997; 26: $1-16$.

36 Carter DA, Bray GM, Aguayo AJ. Regenerated retinal ganglion cell axons can form well-differentiated synapses in the superior colliculus of adult hamsters. J Neurosci 1989; 9: 4042-4050.

37 Brecknell JE et al. Bridge grafts of FGF-4 secreting schwannoma cells promote functional axonal regeneration in the nigrostriatal pathway of the adult rat. Neuroscience 1996; 74: 775-784.
38 Bamber NI et al. Neurotrophins BDNF and NT-3 promote axonal re-entry into the distal host spinal cord through Schwann cell-seeded mini-channels. Eur J Neurosci 2001; 13: $257-268$.

39 Li Y, Field PM, Raisman G. Regeneration of adult rat corticospinal axons induced by transplanted olfactory ensheathing cells. J Neurosci 1998; 18: 10514-10524.

$40 \mathrm{Li} \mathrm{Y,} \mathrm{Field} \mathrm{PM,} \mathrm{Raisman} \mathrm{G.} \mathrm{Repair} \mathrm{of} \mathrm{adult} \mathrm{rat}$ corticospinal tract by transplants of olfactory ensheathing cells. Science 1997; 277: 2000-2002.

41 Ramón-Cueto A, Cordero MI, Santos-Benito FF, Avila J. Functional recovery of paraplegic rats and motor axon regeneration in their spinal cords by olfactory ensheathing glia. Neuron 2000; 25: 425-435.

42 Ramón-Cueto A, Plant GW, Avila J, Bunge MB. Longdistance axonal regeneration in the transected adult rat spinal cord is promoted by olfactory ensheathing glia transplants. J Neurosci 1998; 18: $3803-3815$.

43 Schnell L, Schwab ME. Axonal regeneration in the rat spinal cord produced by an antibody against myelinassociated neurite growth inhibitors. Nature 1990; 343: $69-272$

44 Raineteau O, Fouad K, Noth P, Thallmair M, Schwab ME. Functional switch between motor tracts in the presence of the mAb IN-1 in the adult rat. Proc Natl Acad Sci USA 2001; 98: 6929-6934.

45 Blochlinger S, Weinmann O, Schwab ME, Thallmair M. Neuronal plasticity and formation of new synaptic contacts follow pyramidal lesions and neutralization of Nogo-A: a light and electron microscopic study in the pontine nuclei of adult rats. J Comp Neurol 2001; 433: $426-436$.

46 Huang DW, McKerracher L, Braun PE, David S. A therapeutic vaccine approach to stimulate axon regeneration in the adult mammalian spinal cord. Neuron 1999 ; 24: $639-647$.

47 Bunge RP et al. Observations on the pathology of human spinal cord injury. A review and classification of 22 new cases with details from a case of chronic cord compression with extensive focal demyelination. Adv Neurol 1993; 59: $75-89$.

48 Keirstead HS, Blakemore WF. The role of oligodendrocytes and oligodendrocyte progenitors in CNS remyelination. Adv Exp Med Biol 1999; 468: 183-197.

49 Payne BR, Lomber SG. Reconstructing functional systems after lesions of cerebral cortex. Nat Rev Neurosci 2001; 2: $911-919$.

50 Buonomano DV, Merzenich MM. Cortical plasticity: from synapses to maps. Annu Rev Neurosci 1998; 21: $149-186$.

51 Fawcett JW, Rosser AE, Dunnett SB. Brain Damage and Brain Repair. Oxford University Press, 2000.

52 Romero MI, Rangappa N, Garry MG, Smith GM. Functional regeneration of chronically injured sensory afferents into adult spinal cord after neurotrophin gene therapy. J Neurosci 2001; 21: 8408-8416.

53 Ramer MS, Priestley JV, McMahon SB. Functional regeneration of sensory axons into the adult spinal cord. Nature 2000; 403: $312-316$

54 Murray M, Fischer I. Transplantation and gene therapy: combined approaches for repair of spinal cord injury. Neuroscientist 2001; 7: $28-41$. 